\title{
A special issue on the late Professor Atsushi Komamine
}

\author{
Jang R. Liu $\cdot$ Yang Do Choi
}

Published online: 23 January 2014

(c) Korean Society for Plant Biotechnology and Springer Japan 2014

This Special Issue of Plant Biotechnology Reports was designed to shed light on the contributions to plant biology of the late Professor Atsushi Komamine, a great scientist of our days, who passed away three years ago. Professor Komamine raised significant and fundamental issues on plant biology, and his scholarly career was a quest to answer the questions posed.

In this issue, Professor Hiroaki Kodama sums up the Professor's numerous contributions in the form of a tribute. Professors Masaki Ito, Taku Demura, Tatsuhito Fujimura, Munetaka Sugiyama, and Masaaki Sakuta have written review articles on selected issues which Professor Komamine had raised and continued as his life-time pursuit. These review articles are numbered in the contents as Komamine Review no. 1 through 5 to distinguish them from the regular review articles. This special issue, therefore, will trigger a new high-quality review series from scientists who share the same vision with the late Professor Komamine.

Together with the contributors to this Special Issue, we have not yet done enough to highlight Professor Komamine's scientific insights into, and his vision of, the future of plant biology. We hope that others will help to fill the gaps in the near future.

Finally, we are pleased to announce that, beginning with this issue, Plant Biotechnology Reports will be published six times per year, up from four issues, to accommodate the increased number of high-quality manuscripts that are being submitted.

The Editors-in-Chief,

Jang R. Liu

Yang Do Choi (reviews)

\footnotetext{
J. R. Liu ( $\square)$

Plant Systems Engineering Research Center, Korea Research Institute of Bioscience and Biotechnology, Daejeon 305-806,

Korea

e-mail: jrliu@kribb.re.kr

Y. Do Choi

Department of Agricultural Biotechnology, Seoul National

University, Seoul 151-921, Korea
} 